\title{
SISTEMA DE ESTACIONAMENTO INTELIGENTE PARA AMBIENTE COBERTO: UMA ABORDAGEM BASEADA NA INTERNET DAS COISAS
}

Antonio Ferreira de Oliveira Neto1; Herman A. Lepikson, Dr.Eng..; Mário Augusto Santana de Oliveira Júnior ${ }^{1}$; Rafael Barbosa Mendes².

${ }^{1}$ Programa de Pós-Graduação em Mecatrônica, UFBA; Salvador/Bahia; antoniofon14@gmail.com

${ }^{2}$ Programa de Pós-Graduação em Gestão e Tecnologia, Senai Cimatec; Salvador/Bahia;

Resumo: Sistemas inteligentes para estacionamentos cobertos buscam dinamizar e acelerar a procura por vagas e veículos em um ambiente. Este estudo visa identificar como estão sendo direcionadas as pesquisas em relação ao desenvolvimento dos sistemas para estacionamentos inteligentes com o uso da internet das coisas. Realizou-se busca, em quatro bases de dados nacionais e estrangeiras para a caracterização da revisão de literatura. Como resultado da busca, 40 artigos e trabalhos foram selecionados, realizando-se avaliação criteriosa quanto à relevância e às tecnologias empregadas na construção dos sistemas. Assim, conclui-se que existem publicações sobre sistemas inteligentes que facilitem a procura por vagas, mas poucas que tratam também da localização de veículos em um estacionamento.

Palavras-Chave: Estacionamentos inteligentes; Ambientes cobertos; Internet das Coisas.

\section{SMART PARKING SYSTEM FOR ENVIRONMENT INDOOR: AN APPROACH BASED IN INTERNET OF THINGS}

\begin{abstract}
Smart systems to parking indoor seek streamline and accelerate search for spaces and vehicles in an environment. This study aims to identify how research is being directed towards the development of smart parking systems using the internet of things. A search was performed in four national and international databases to characterize the literature review. As a result of the search, 40 articles and works were selected, where a careful evaluation was carried out as to the relevance and technology used in the systems development. Thus, it is concluded that there are publications on intelligent systems that facilitate the search for parking spaces and vehicles, but still few that also address the location of vehicles in a parking lot.
\end{abstract}

Keywords: Smart parking; Indoor environments; Internet of things. 


\section{INTRODUÇÃO}

A alteração no ritmo de vida e nas basilares estruturas econômicas e sociais promovem uma maior dinâmica à vida da população. As tecnologias desenvolvidas para melhoria da comunicação e transferência de informação são de extrema importância e decisivas para a aceleração das mudanças em uma sociedade informatizada e em desenvolvimento [1]. A dinâmica atual de vida dos cidadãos não é tão restrita à convivência doméstica, como em tempos passados. Nos dias atuais as pessoas têm um ritmo mais acelerado que envolve viagens diárias para trabalhar, estudar e realizar atividades de cunho turístico ou de consumo.

O deslocamento dos cidadãos que utilizam automóveis é bastante simplificado e facilitado quando há a utilização do navegador com aporte de um sistema de posicionamento global (GPS), que geralmente é utilizado para navegação rodoviária ou trafegar nas ruas de uma cidade. Mesmo com a utilização de GPS para a navegação, a busca de vagas para estacionar é bastante limitada e demorada pelo fato de este recurso não ser projetado para tanto. A busca por vaga pelos meios convencionais gera perdas de tempo e para o meio ambiente, que é comprometido pelo lançamento de gases tóxicos na atmosfera. Hoje os sistemas de estacionamento a céu aberto contam com o auxílio de algumas tecnologias de comunicação e informação (TIC) que melhoram as condições para um usuário, por meio do desenvolvimento de aplicações particulares e informativos por quadrante de estacionamento [2]. O mesmo não se pode afirmar quando se trata de estacionamentos fechados.

Quando se trata de navegação em ambientes cobertos ou fechados como são as situações encontradas em estacionamentos de shoppings, hospitais e prédios, nota-se que as navegações por GPS e outros dispositivos são bastante comprometidas, devido a fatores que são intrínsecos das estruturas construtivas dos ambientes, que podem causar interferência. Isto dificulta a vida dos motoristas e pilotos que necessitam utilizar estacionamentos nesses locais [3].

Os sistemas de estacionamentos desenvolvidos na atualidade estão ganhando uma quantidade maior de possibilidades tecnológicas, processo que melhora a confiabilidade dos sistemas e melhora a precisão das informações embarcadas pelo desenvolvedor [1]. Com isso, os utilizadores de serviços baseados em sistemas inteligentes desenvolvidos diretamente para facilitar a vida na busca por vagas e veículos em estacionamentos ganham um tempo valioso para o dia-a-dia, sendo possibilitada uma maior eficácia na distribuição de serviços de estacionamento.

Para [4] a vinculação dos serviços de estacionamento inteligentes à internet das coisas melhora as condições para a geração de sistemas com maior eficácia e precisão, durante a aquisição, envio e recepção de dados que são disponibilizados à nuvem por meio dos instrumentos ligados a rede de identificação veicular em um estacionamento. Isso possibilita disponibilizar e melhorar as condições estabelecidas pelas redes desenvolvidas para estacionamentos inteligentes, de modo a possibilitar o gerenciamento, navegação e informação tanto para proprietários comerciais quanto para os clientes, que podem contar com serviços de informação a segurança veicular, localização e pagamento do serviço prestado pelo estacionamento. 
Desse modo, o grande desafio para a inserção de sistemas inteligentes direcionados a estacionamento está na melhoria do desempenho dos navegadores para ambientes cobertos, aprimoramento dos sinais captáveis para esses ambientes, rede de sensores adaptativa e redução da interferência humana nos sistemas para estacionamentos.

\section{METODOLOGIA ADOTADA}

O uso de plataformas de buscas de trabalhos acadêmicos foi utilizado para direcionar a escolha dos trabalhos cujos temas estão em consonância com a utilização de sistemas de estacionamento inteligente vinculados à abordagem de internet das coisas, com o uso das palavras chaves em inglês e em português mostradas na figura 1. As principais bases de dados utilizadas foram o Google Acadêmico, ScienceDirect da Elsevier, SciELO - Scientific Electronic Library Online e IEEE Xplorer Digital Library.

Figura 1. Palavras-chave da pesquisa em inglês e português. Elaborado pelos autores.

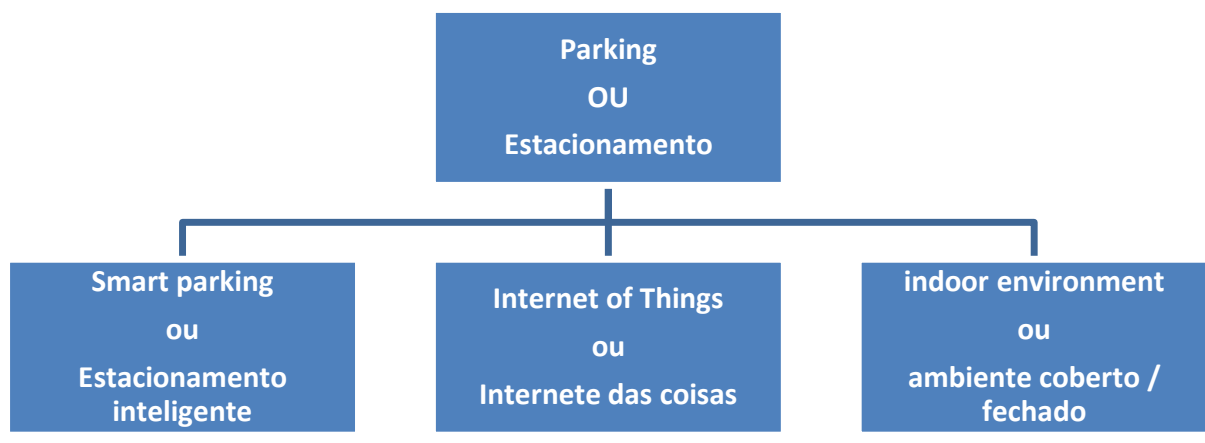

O desenvolvimento de critérios de avaliação para a escolha dos artigos que tornam viável à conclusão da pesquisa tornam-se necessários para auxiliar a exclusão de trabalhos que possuíssem características de desenvolvimento diferentes das pesquisadas, tempo igual ou menor a 9 anos de publicação e que utilizasse ao menos duas das palavras chaves designadas na pesquisa em seu desenvolvimento. A tabela 1 abaixo mostra o quantitativo de artigos encontrados em cada ano descrito e o número final de pesquisas selecionadas para o desenvolvimento do trabalho.

Tabela 1. Base de seleção de trabalhos correlatos. Elaborado pelos autores.

\begin{tabular}{|l|c|c|c|}
\hline & $\begin{array}{c}\text { Antigos } \\
\text { encontrados }\end{array}$ & Pré-selecionados & Selecionados \\
\hline $\mathbf{2 0 1 9}$ & 8 & 3 & 3 \\
\hline $\mathbf{2 0 1 8}$ & 7 & 1 & 1 \\
\hline
\end{tabular}




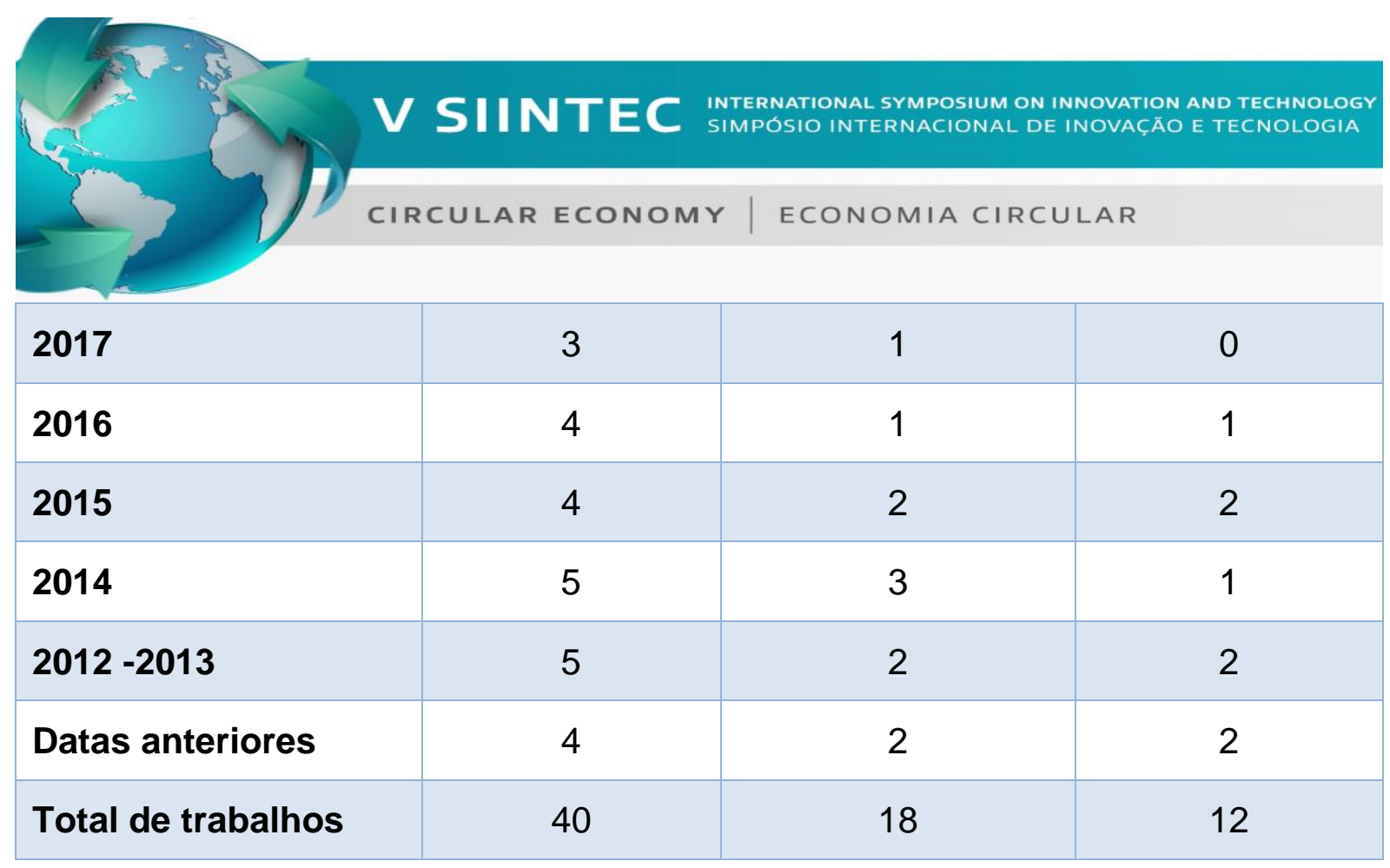

\section{RESULTADOS}

A busca por artigos relacionados ao tema em desenvolvimento resultou na seleção de 40 artigos e trabalhos. Em uma primeira etapa de seleção 18 artigos trabalhavam nos segmentos de desenvolvimento de projetos com utilização da internet das coisas (em inglês, Internet Of Things- IOT) para estacionamentos inteligentes. Desses, apenas 12 utilizam seus estudos voltados a implantação de projetos para ambientes cobertos ou fechados.

Desse modo, será discutido no subitem a seguir as tecnologias e arquiteturas utilizadas para o desenvolvimento dos trabalhos, processo que poderá estabelecer como estão sendo desenvolvidas as pesquisas e projetos para estacionamentos inteligentes.

\subsection{Discussão dos Trabalhos Selecionados}

A realidade do desenvolvimento de projetos pensando na acomodação de veículos em vagas de estacionamento e na comodidade para o usuário que busca uma vaga já era uma preocupação desde o fim da década de 90 para o início dos anos 2000, período em que [5] patenteou um projeto que utilizava sensores e antenas de rádio frequência para comunicação e transmissão de dados para o gerenciamento de vagas em um estacionamento, com a tecnologia de Sistema de Posicionamento Global (em inglês, Global Positioning System - GPS).

No trabalho de [6] é realizada a implementação de um sistema de gerenciamento de estacionamento em que há a alocação das informações em um banco de dados, de modo a incrementar ao sistema de gerenciameno a possibilidade de encontrar vagas de estacionamento vazias, cobrança automática de taxas de estacionamento, gerenciamento de segurança e relatório estatístico, por meio da 
utilização da arquitetura formada por rede de sensores, MOTE-VIEW, banco de dados PosgreSQL, TinyOS, Banco de dados CarRecord e o aplicativo de estacionamento.

$\mathrm{Na}$ busca por melhorias nos sistemas de estacionamentos inteligentes, [1] desenvolve condições importantes para melhorar os parâmetros de comodidade para o cliente de um estabelecimento comercial onde o estacionamento é coberto e dificulta a utilização do GPS para navegação. A integração do sistema de GPS para navegação rodoviária com a navegação para ambiente interno, que utilizam mapas de piso baseados em imagens, é realizado por meio de uma solução de posicionamento precisa denominada HIPE, que utiliza a leitura dos sensores adquirida pela interface de programação de aplicativos QtMobility para indicar se há ou não vaga disponível. Os dados dos sensores são lançados na base de dados que está conectada via Wireless Local Area Network (WLAN, em português, rede local sem fios) e disponibiliza o recurso para os usuários via aplicativo de celular que, por meio de sistema de navegação próprio, é capaz de encontrar uma vaga previamente selecionada no aplicativo e posteriormente reencontrar o veículo.

Já no trabalho desenvolvido por [7] há, junto ao sistema inteligente do estacionamento, a utilização de comunicação via SMS para que, na chegada ao estacionamento, o usuário digitando o número de seu telefone celular, é designado um local onde o veículo será estacionado e um mapa é mostrado na tela, sendo enviada informação do local a ser estacionado o veículo para o celular do condutor. Esse processo pode ser realizado, pois o estacionamento está equipado com uma estação supervisora central com modem GSM e cada local de estacionamento com um sensor luminoso para detectar se há ou não veículo estacionado.

Na pesquisa desenvolvida por [8] são utilizados sensor ultrassom para detectar a vaga de estacionamento, rede sem fio nRF24L01+ para a transmissão e recepção de radiofrequências e processador Raspberry $\mathrm{Pi}$ com o intuito de indicar, por meio de um aplicativo, se há vaga disponível ou não em um estacionamento. [9] por sua vez, busca a integração de sistemas multiagentes para a construção de seu projeto que utiliza a plataforma Jacamo para alocação de vagas em estacionamento inteligente, capaz de integrar o sistema do estacionamento com o usuário, informando o local a ser estacionado através da solicitação do usuário.

Com outra perspectiva, [10] gera um sistema capaz de identificar a quantidade de vagas ocupadas e disponíveis. A leitura de etiquetas RFID gera uma contagem de veículos entrando e saindo de um estacionamento, lançando os dados na nuvem por meio de sensores de rede sem fio ZlgBee, processo que disponibiliza através de cálculos a quantidade de vagas disponíveis em cada parque de estacionamento. Já [11], em uma mesma linha de trabalho que [9], incorpora ao sistema a utilização de sensores ultrassônicos conectados a esp8266s e beacons instalados em cada esquina do estacionamento, além de gerar mapas internos para navegação do cliente até uma das vagas, finalizando aí o processo de navegação.

Para a realização do trabalho de [12] há a instalação de sensores de dois tipos associados ao Bluetooth Low Energy (BLE) para envio, recepção e comunicação com os usuários. Em ambientes internos são instalados sensores ultrassônicos em 
conjunto com os BLE em cada vaga e no ambiente externo há a utilização de sensores magnéticos também instalados em conjunto com os BLE. Os dados desses sensores são enviados para o Módulo de Identidade Universal do Assinante, junto com a identificação de cada usuário para o servidor através de Zigbee com gateway. Este processo possibilita ao servidor identificar o local de estacionamento e consequentemente o veículo.

No sistema de estacionamento planejado por [13], os usuários são informados, na entrada do estacionamento, em qual lugar está posicionada a vaga mais próxima de acordo com o tamanho do veículo e o direcionamento é mostrado após simulação do sistema Proteus com a utilização do algoritmo de Dijkstra.

Os trabalhos desenvolvidos por $[14,15]$ seguem a linha de identificação de um usuário, indicação de ocupação ou disponibilidade de uma vaga em um estacionamento baseada em sensores fixos e, por fim, conectividade com sistemas sem fio para a acomodação dos usuários nas vagas. Não se fornece informações adicionais quanto à navegação interna dos ambientes, apenas utilizando sinalização luminosa.

\section{CONCLUSÃO}

Apesar do grande número de tecnologias desenvolvidas passiveis de utilização para o aprimoramento dos sistemas de estacionamento inteligente, conclui-se que ainda existem obstáculos a serem superados no desenvolvimento de sistemas complexos e que possam gerar produtos com maior qualidade e confiabilidade para os usuários, a custos viáveis.

Desse modo, tecnologias como câmeras de alta resolução e outros sensores e formas de envio de informação podem ser implantadas em conjunto com módulos sensores para possibilitar maior comodidade e possibilidade de escolha para os usuários de estacionamentos fechados ou cobertos no processo de identificação do local em que seus veículos estão estacionados, bem como estacioná-los mais rapidamente, minimizando o esforço e diminuindo o processo poluidor gerado pela queima desnecessário de combustíveis.

\section{Agradecimentos}

O presente trabalho foi realizado com apoio da Coordenação de Aperfeiçoamento de Pessoal de Nível Superior - Brasil (CAPES) - Código de Financiamento 001 e dos professores do curso de Pós-graduação em Mecatrônica da Universidade Federal da Bahia. 


\section{REFERÊNCIAS}

${ }^{1}$ LIU, Jingbin et al. iParking: An intelligent indoor location-based smartphone parking service. Sensors, v. 12, n. 11, p. 14612-14629, 2012.

${ }^{2}$ IDRIS, M. Y. I. et al. Car park system: a review of smart parking system and its technology. Information Technology Journal, v. 8, n. 2, p. 101-113, 2009.

${ }^{3}$ FERNANDES, Hugo et al. Location based services for the blind supported by RFID technology. Procedia Computer Science, v. 27, p. 2-8, 2014.

4 KHANNA, Abhirup; ANAND, Rishi. IoT based smart parking system. In: 2016 International Conference on Internet of Things and Applications (IOTA). IEEE, p. 266-270, 2016.

${ }^{5}$ CLAPPER, Edward O. Method and apparatus for parking management system for locating available parking space. U.S. Patent n. 6,147,624, 14 nov. 2000.

${ }^{6}$ TANG, Vanessa WS; ZHENG, Yuan; CAO, Jiannong. An intelligent car park management system based on wireless sensor networks. In: 2006 First International Symposium on Pervasive Computing and Applications. IEEE, p. 65-70, 2006.

7 JEFFREY, Joseph et al. Smart parking system using wireless sensor networks. In: The Sixth International Conference on Sensor Technologies and Applications. p. 306-311, 2012.

${ }^{8}$ CAVAMURA, Humberto Fernando Massaharu; MITSUHASHI, Marlos Kenjy. Sistema de gerência de vagas de estacionamento. 2014. Trabalho de Conclusão de Curso. Universidade Tecnológica Federal do Paraná.

${ }^{9}$ CASTRO, Lucas Fernando Souza de. Modelagem e implementação de um sistema multiagente utilizando a plataforma Jacamo para alocação de vagas em um estacionamento inteligente. 2015. Trabalho de Conclusão de Curso. Universidade Tecnológica Federal do Paraná.

10 PHAM, Thanh Nam et al. A cloud-based smart-parking system based on Internet- 
of-Things technologies. IEEE Access, v. 3, p. 1581-1591, 2015.

11 TSAI, Ming-Fong; KIONG, Ye Chin; SINN, Ang. Smart service relying on Internet of Things technology in parking systems. The Journal of Supercomputing, v. 74, n. 9, p. 4315-4338, 2018.

12 LEE, Chungsan et al. Smart parking system for Internet of Things. In: 2016 IEEE International Conference on Consumer Electronics (ICCE). IEEE, p. 263-264, 2016.

13 ATA, KI Mohammad et al. Smart Indoor Parking System Based on Dijkstra's Algorithm. International Journal of Electrical Engineering and Applied Sciences (IJEEAS), v. 2, n. 1, p. 13-20, 2019.

14 DESHMUKH, Abhay; JOSHI, Radhika D. UNDERSTANDING THE ARCHITECTURE OF INTERNET OF THINGS USING A CASE STUDY OF SMART PARKING b. Asian Journal For Convergence In Technology (AJCT), 2019.

15 BALHWAN, Suman et al. Smart Parking-A Wireless Sensor Networks Application Using loT. In: Proceedings of 2nd International Conference on Communication, Computing and Networking. Springer, Singapore, p. 217-230, 2019. 